\title{
Investigación translacional para generar datos a favor de la introducción racional y eficiente de vacunas nuevas en países en vías de desarrollo: La experiencia del Instituto Internacional de Vacunas
}

\author{
John Clemens \\ Instituto Internacional de Vacunas, Seúl, Corea
}

\author{
Palabras clave \\ Investigación translacional • Introducción de vacunas • \\ Países en vías de desarrollo
}

\section{Extracto}

En los últimos años, la introducción de vacunas nuevas en países opulentos ha tenido lugar a un ritmo asombroso. En comparación, se han introducido pocas vacunas de nueva generación en los programas de salud pública para los pobres de los países en vías de desarrollo, y para aquéllos que sí los tienen, la introducción ha sido penosamente lenta. Los recursos financieros limitados han retrasado la introducción. La lentitud también se debe a una escasez de datos necesarios para adoptar decisiones políticas racionales. El apoyo inadecuado para realizar estudios de fase 1 con candidatos vacunales orientados a enfermedades en países en vías de desarrollo es un obstáculo bien conocido. Además, se precisan otros tipos de investigación translacional para generar las pruebas necesarias para establecer una política. Se ha observado que numerosas vacunas actúan con menos eficacia en poblaciones empobrecidas del mundo en fase de desarrollo que en personas que residen en países más opulentos. En consecuencia, los ensayos de fase 2 y fase 3 constituyen un segundo tipo esencial de investigación translacional necesaria para la introducción de vacunas en países en vías de desarrollo. Por otra parte, incluso para las vacunas que obtienen la aprobación a través de ensayos clínicos en países en vías de desarrollo, pueden persistir dudas acerca de si la carga de patología justifica la introducción de la vacuna, si la administración de la vacuna en programas sanitarios será rentable, si la introducción de la vacuna será programáticamente factible y aceptable y si la introducción de la vacuna será financieramente sostenible. Para abordar estas dudas residuales es imprescindible un tercer tipo de investigación translacional. Desde sus comienzos, en 1987, el Instituto Internacional de Vacunas, una organización de investigación y desarrollo internacional sin afán de lucro, ubicada en Seúl, Corea, ha realizado investigación translacional con la introducción de nuevas vacunas para muy diversas enfermedades en 22 países de Asia, África, y América Latina. En este trabajo describimos los proyectos de investigación translacional emprendidos por el Instituto Internacional de Vacunas y las lecciones aprendidas sobre estrategias para incrementar el impacto de la investigación translacional sobre la política vacunal para el mundo en vías de desarrollo.

Copyright $\odot 2008$ Nestec Ltd., Vevey/S. Karger AG, Basel

\section{Introducción}

Vivimos en medio de una revolución en el desarrollo de vacunas, en la cual se han elaborado y aprobado vacunas a un ritmo sin precedentes y se han aplicado nuevas tecnologías referidas al desarrollo de nuevas vacunas, incluyendo aquéllas contra enfermedades que en el pasado no se consideraban evitables mediante vacunas. A pesar de esto, el ritmo de introducción de vacunas nuevas y perfeccionadas en los programas sanitarios de países en vías de desarrollo ha sido penosamente lento [1]. Esto es tanto

\section{KARGER}

Fax +4161306 1234 E-Mail karger@karger.ch www.karger.com
(C) 2008 Nestec Ltd., Vevey/S. Karger AG, Basel 0252-8185/08/0662-0081\$24.50/0

Accesible online en:

www.karger.com/ans
John Clemens, MD

International Vaccine Institute

Kwanak PO Box 14

Seoul 151-600 (Korea)

Tel. +82 2881 1011, Fax +82 2872 2803, E-Mail jclemens@ivi.int 
más desafortunado cuanto que muchas de esas vacunas de nueva generación, como las diseñadas contra la diarrea, la meningitis y la neumonía, están orientadas contra enfermedades que constituyen los problemas sanitarios más acuciantes en los países en vías de desarrollo.

Mucho se ha escrito sobre la problemática científica del descubrimiento de las vacunas y sobre las estrategias para mejorar el proceso de descubrimiento de nuevos candidatos vacunales. También se conocen perfectamente los escollos financieros y los obstáculos programáticos que impiden la introducción de vacunas nuevas en países en vías de desarrollo. Menos valorado es el hecho de que, incluso para candidatos vacunales existentes, pueden existir otros retos científicos formidables capaces de impedir su introducción en los programas sanitarios. El término 'investigación translacional' abarca la agenda de investigación que aborda estos retos. Como tal, la investigación translacional puede considerarse una investigación para transformar tanto los candidatos vacunales experimentales como las vacunas ya aprobadas en herramientas prácticas que son utilizadas en los procedimientos de salud pública.

La investigación translacional ha sido un enfoque muy importante del Instituto Internacional de Vacunas (IIV) en Seúl, Corea. El IIV comenzó al principio de los años 90 como un proyecto germinal del Programa de Desarrollo de Naciones Unidas. A la sazón, el Programa de Desarrollo de Naciones Unidas consideraba que el mundo necesitaba una organización de investigación internacional dedicada exclusivamente a desarrollar y desplegar vacunas nuevas para países en vías de desarrollo. En 1997, el IIV llegó a ser una organización internacional autónoma bajo la Convención de Viena que actualmente cuenta con 40 países y la Organización Mundial de la Salud como firmantes de su carta constitucional, y con la República de Corea como país anfitrión. Si bien ha dejado de ser una organización de la ONU, el IIV sigue siendo un miembro de la familia de organizaciones de la ONU. En este trabajo perfilamos de entrada los tipos de estudios que recaen bajo la rúbrica de la investigación translacional y, seguidamente, ilustramos estos estudios con el trabajo realizado por el IIV durante los últimos años.

\section{El paradigma clínico para evaluar vacunas destinadas a su aprobación}

Para situar dentro de su contexto el fundamento de la investigación translacional, es útil describir las fases de la evaluación clínica de los candidatos vacunales antes de la aprobación [2,3]. Los estudios humanos iniciales de un candidato que ha generado previamente resultados prometedores en los estudios preclínicos se denominan ensayos de fase 1. Estos ensayos son normalmente de tamaño reducido (por ejemplo, incluyen aproximadamente de 10 a 20 sujetos adultos sanos) y están diseñados fundamentalmente para evaluar si la vacuna se asocia a efectos adversos frecuentes. Los ensayos de fase 1 evalúan normalmente otros efectos de las vacunas, como las respuestas inmunitarias y la excreción de la vacuna (en caso de candidatos vacunales atenuados) y pueden utilizarse para determinar la dosis y la pauta óptimas para el candidato vacunal.

Los candidatos considerados aptos en los ensayos de fase 1 pueden seguir investigándose en los ensayos de fase 2. Estos se distinguen por el tamaño más amplio de la muestra (frecuentemente en un orden de varios centenares de sujetos), por su capacidad para evaluar, de un modo estadísticamente significativo, las respuestas inmunitarias, así como las reacciones adversas relativamente infrecuentes y, en último lugar, por la inclusión de sujetos de las poblaciones y los grupos de edad que serán los destinatarios finales de la vacuna en los procedimientos de salud pública. Además, los ensayos de fase 2 pueden medir la excreción y la transmisibilidad de las vacunas atenuadas. Aunque los ensayos de fase 2 no se diseñan normalmente para medir la protección de la vacuna frente a la infección seleccionada, se realizan tipos especiales de ensayos de fase 2, en ocasiones denominados 'ensayos de fase $2 \mathrm{~b}$, para medir el efecto protector de la vacuna [4]. Los ensayos de fase $2 b$ corresponden a dos tipos diferentes de estudios. En el primero se mide la protección de la vacuna frente a provocaciones experimentales con el agente patógeno seleccionado. En el segundo, se mide la protección vacunal en condiciones de campo, aunque con tamaños de la muestra menores que los normalmente necesarios en los ensayos de fase 3. Ambos tipos de estudios de fase $2 b$ se emprenden para realizar una comprobación del principio de que la vacuna es capaz de proteger frente a la enfermedad en humanos antes de invertir en ensayos de fase 3 , más costosos y logísticamente más exigentes.

Por último, para candidatos hallados adecuadamente seguros e inmunógenos en los ensayos de fase 2 pueden diseñarse ensayos de fase 3 . El papel distintivo de los ensayos de fase 3 es que miden la protección de la vacuna frente a infecciones de presentación natural en poblaciones de riesgo. Los ensayos de fase 3 suelen ser bastante extensos, a menudo incluyen miles de sujetos, y siempre se realizan en las poblaciones y los grupos de edad que son destinatarios de la vacuna. Debido a su tamaño im- 
portante, los ensayos de fase 3 suelen ser capaces de detectar efectos adversos más bien infrecuentes asociados a la vacunación. Por otra parte, dado que los ensayos de fase 3 miden tanto las respuestas inmunitarias a la vacunación como la protección vacunal, pueden llegar a correlacionar ambos elementos $y$, de este modo, derivar correlatos inmunológicos de protección. Los ensayos de fase 1 pueden realizarse de forma controlada o no controlada; convencionalmente, los ensayos de fase 2 y fase 3 se basan en diseños a doble ciego, aleatorizados y controlados.

\section{Investigación translacional: Superación de obstáculos a la introducción de nuevas vacunas}

La investigación translacional para acelerar la introducción de un candidato vacunal en programas sanitarios de países en vías de desarrollo aborda tres tipos de problemas científicos. Primero, un candidato vacunal puede languidecer en las estanterías del laboratorio sin tener ninguna oportunidad de ser investigado en humanos. Este escollo al desarrollo es más acusado en el caso de vacunas sin interés comercial, orientadas a enfermedades de escaso o nulo interés para poblaciones de países industrializados y cuyo provecho potencial es limitado para los productores de vacunas. Entre los ejemplos de tales vacunas destacaremos las dirigidas contra la leishmaniasis, la enfermedad por uncinaria y la esquistosomiasis. Dado que estas enfermedades afectan fundamentalmente a países en vías de desarrollo y que los viajeros adinerados que se dirigen a dichos países no presentan un riesgo particularmente elevado de contraerlas, el incentivo económico por parte de la industria para emprender programas costosos de desarrollo clínico es escaso.

En segundo lugar, incluso para vacunas que son de interés tanto para poblaciones de países industrializados como para países menos desarrollados, el interés y los recursos pueden centrarse únicamente en estudios humanos realizados en países industrializados, que es donde se localizan los mercados más rentables. La puesta en práctica de vías de desarrollo clínico paralelas en países industrializados y países en vías de desarrollo puede imponer riesgos de retrasos considerables en la aprobación en los mercados de los países industrializados, con pérdidas importantes en la generación de ingresos para la industria. Esto crea un problema para los países en vías de desarrollo, dado que los resultados de los estudios efectuados en poblaciones de países industrializados no siempre pronostican el comportamiento de una vacuna en pobla- ciones de países en vías de desarrollo. Este ha sido el caso de las vacunas de primera generación antirotavirus [5-7] y contra Haemophilus influenzae de tipo b (Hib) [8-10], que producen un notable efecto protector en niños de países industrializados pero un efecto protector precario en niños de países en vías de desarrollo. En el pasado, con demasiada frecuencia se realizaron estudios en países en vías de desarrollo muchos años después de la aprobación de una vacuna en países industrializados, generando un retraso inaceptable en la introducción de la vacuna incluso en casos en que los ensayos habían mostrado resultados favorables.

En tercer lugar, a pesar de haberse demostrado que una vacuna produce efectos seguros y protectores en ensayos de fase 3 en países en vías de desarrollo, los responsables de la formulación de políticas pueden seguir albergando dudas sobre si resultará apropiado introducir la vacuna en los programas sanitarios de sus países. Esto es debido a que los datos proporcionados por las evaluaciones previas a la aprobación, incluso las realizadas en los países en vías de desarrollo, no son normalmente capaces de abordar numerosas preguntas prácticas sobre la introducción de una vacuna nueva en programas de la vida real, preguntas que deben de ser respondidas antes de adoptar una decisión encaminada a la introducción de una vacuna $[11,12]$. La evidencia insuficiente en la investigación aguas abajo requerida constituye el tercer escollo científico.

La superación de estos tres obstáculos exige tres tipos de investigación translacional [11-13]. El primer escollo científico requiere la realización de estudios humanos iniciales (fase 1) con candidatos vacunales prometedores. El segundo exige la realización de estudios de fase 2 y fase 3 para obtener la aprobación de la vacuna en ámbitos de países en vías de desarrollo, realizados en condiciones ideales paralelamente con evaluaciones en países industrializados. Por último, el tercer escollo requiere, para vacunas que han demostrado seguridad y eficacia en los ensayos de fase 3 en el mundo en vías de desarrollo, la generación de pruebas epidemiológicas, clínicas, económicas, conductuales y políticas, necesarias para asegurarse de que la introducción de una vacuna nueva en los programas de salud pública de un país en vías de desarrollo es racional, factible, aceptable y asequible.

En la tabla 1 se presentan las prioridades de enfermedades actuales para la investigación translacional del IIV sobre vacunas de nueva generación; por su parte, la figura 1 ilustra un cuadro geográfico de los países asociados al IIV para la realización de sus programas de investigación translacional. En el resto de este trabajo proporcio- 


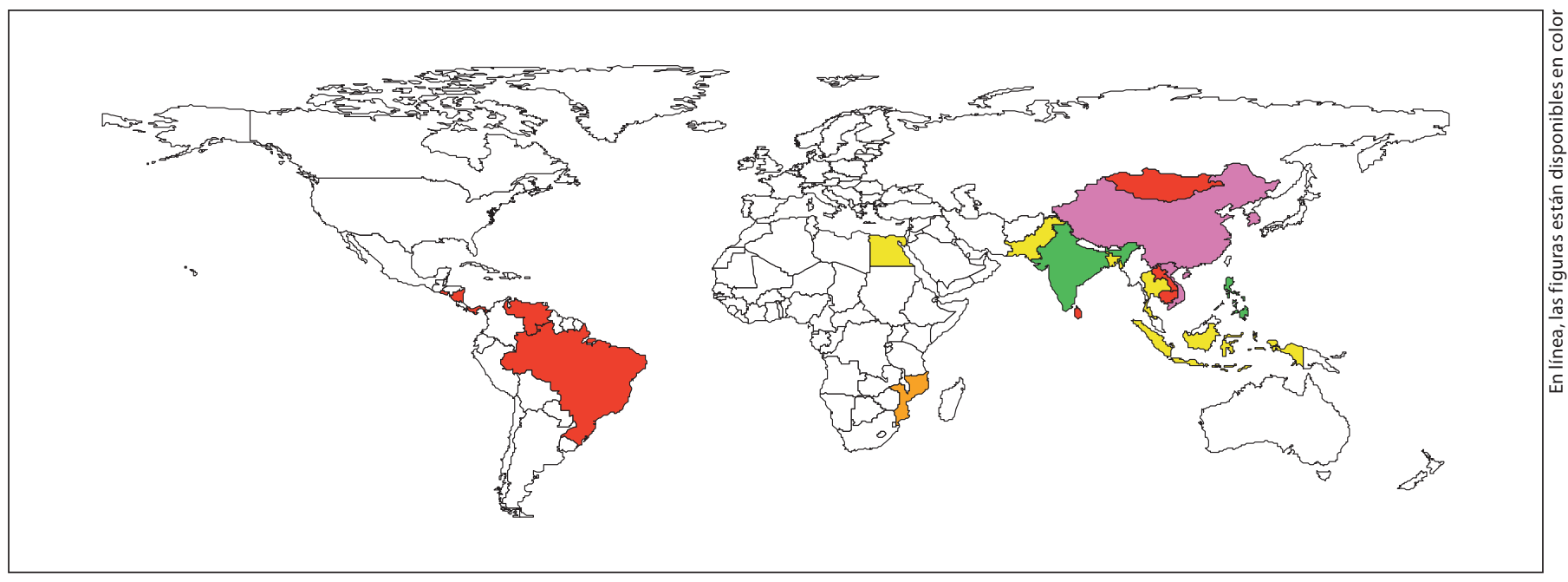

Fig. 1. Localizaciones geográficas de los programas de investigación translacional del IIV.

Tabla 1. Prioridades de la investigación translacional del IIV

1. Vacunas contra infecciones entéricas

Rotavirus

Escherichia coli enterotoxígena

Shigellosis

Cólera

Fiebre tifoidea

2. Vacunas contra causas de meningitis y/o neumonía

$\mathrm{Hib}$

Neumococo

Meningococo

Gripe

Tuberculosis

3. Vacunas contra infecciones por flavivirus EJ

Fiebre Dengue

namos ejemplos de los programas de investigación translacional del IIV para ilustrar cómo se ha emprendido la investigación para abordar la problemática de superación de dichos escollos.

\section{Investigación translacional de vacunas antes de la aprobación: Asociaciones para asegurar el acceso a las vacunas}

La generación y la comunicación de los datos obtenidos por la investigación translacional son cruciales para adoptar decisiones políticas racionales sobre la introduc- ción de vacunas y pueden actuar como un mecanismo de 'tirón' para crear demanda de una vacuna y recursos para su adquisición [12]. Sin embargo, esta investigación no ejercerá ningún impacto sobre la introducción de la vacuna a menos que se asocie a estrategias que permitan disponer de la vacuna seleccionada en cantidades suficientes y a precios rentables. Las asociaciones públicasprivadas entre entidades sin afán de lucro, como el IIV, y los fabricantes de vacunas han llegado a ser un mecanismo corriente por el cual los riesgos del desarrollo de vacunas antes de la aprobación son compartidos por los sectores público y privado, pudiendo el sector público beneficiarse de este modo al obtener la seguridad de un suministro amplio y rentable de la vacuna cuando la vacuna sea efectivamente aprobada.

El IIV ha buscado varios modelos diferentes para dichas colaboraciones, todos ellos realizados dentro de los programas de investigación translacional. Todos estos modelos suponen trabajar con productores de vacunas comprometidos en poner una vacuna a disposición en cantidades suficientes y a precios rentables después de obtener su aprobación. Uno de los modelos se basa en la colaboración con compañías farmacéuticas internacionales muy importantes. Por ejemplo, el IIV colabora actualmente con Merck Vaccines en un ensayo de fase 3 con una vacuna contra el rotavirus oral atenuada (Rotateq ${ }^{\mathrm{TM}}$ ). Esta vacuna ha sido aprobada basándose en una serie extensa de ensayos clínicos realizados en países industrializados [14]. No obstante, dado que las vacunas orales atenuadas contra el rotavirus y otros agentes patógenos han actuado menos favorablemente en niños de países en vías 
de desarrollo, la Alianza GAVI (el principal procurador de vacunas para niños de países en vías de desarrollo) adquirirá esta vacuna para estos países sólo después de haber obtenido datos sobre su eficacia en ensayos clínicos realizados en el mundo en vías de desarrollo. Con el patrocinio del Programa de Vacunas Antirotavirus en el PATH (Programa de Tecnología Apropiada en Sanidad) y Merck, el IIV está colaborando actualmente con el Instituto Nacional de Higiene y Epidemiología de Vietnam en un ensayo de eficacia de fase 3 con esta vacuna en Nha Trang, Vietnam. Este ensayo complementará a otros varios que se están realizando en países en vías de desarrollo de Asia y África.

En un segundo modelo de colaboración se está trabajando con un candidato vacunal que es actualmente propiedad de una compañía de biotecnología en el mundo industrializado, con objeto de ultimar la transferencia de tecnología a una compañía capaz de producir la vacuna a gran escala y a precios asequibles. Un ejemplo de una vacuna que está siendo evaluada en este tipo de colaboración es Perú-15, una vacuna contra el cólera oral, genéticamente atenuada, desarrollada originalmente en la Universidad de Harvard y transferida subsiguientemente bajo licencia a Avant Immunotherapeutics. A pesar de los resultados prometedores obtenidos en voluntarios norteamericanos, la vacuna languideció durante años sin ser evaluada en un país donde el cólera es endémico. En el año 2000, el IIV lanzó el programa Enfermedades de los más Empobrecidos (DOMI), financiado por la Fundación Bill y Melinda Gates, para acelerar la introducción de vacunas de nueva generación contra la fiebre tifoidea, el cólera y la shigellosis. Como componente del programa DOMI, el IIV lanzó una serie de ensayos de fase 2 con Perú-15 en Bangladesh, en colaboración con investigadores del Centro Internacional para la Investigación de Enfermedades Diarreicas, Bangladesh. En los ensayos se demostraron la rotunda seguridad e inmunogenicidad de una dosis única de Perú-15 en adultos, niños y lactantes de Bangladesh [15, 16]. Basándose en estos resultados prometedores y con la financiación adicional de la Fundación Gates, el IIV está coordinando actualmente un programa de desarrollo clínico con esta vacuna tanto en India como en Bangladesh en colaboración con el Instituto Nacional de Cólera y Enfermedades Entéricas y el Centro Internacional para la Investigación de Enfermedades Diarreicas, Bangladesh, respectivamente. Este programa incluye ensayos de fase 2 adicionales, conducentes a un extenso ensayo de fase 3 que será realizado en Bangladesh. Si el ensayo de fase 3 proporcionase resultados satisfactorios, el IIV ayudaría a Avant con respecto a la transferencia de la vacuna a un productor de vacunas calificado en un país donde el cólera es endémico.

Otro ejemplo de segundo modelo se refiere al candidato vacunal oral atenuado contra la fiebre tifoidea, $\mathrm{ZH} 09$, que ha generado resultados muy prometedores como vacuna de dosis única en estudios en pacientes hospitalizados y pacientes ambulatorios realizado en EE.UU. y RU [17]. Debido a estos resultados alentadores, el IIV colaboró con Europa Emergente en RU, el elaborador de esta vacuna, y la Unidad de Enfermedades Tropicales de Wellcome en la ciudad Ho Chi Minh, en estudios de fase 2 con esta vacuna en una población endémica de fiebre tifoidea en Vietnam. Estudios realizados en adultos y niños de edad escolar revelaron que la seguridad y la inmunogenicidad de la vacuna eran aceptables, por lo que actualmente se está planificando la realización de estudios de fase 2 adicionales y un ensayo de eficacia de fase 3 en Kolkata, India. Igual que en el caso de la vacuna Perú-15, el IIV también colaborará en la transferencia de la tecnología de producción de esta vacuna a un productor en un país donde la fiebre tifoidea es endémica, de manera que la vacuna pueda al final estar disponible a precios asequibles para programas de salud pública.

Un tercer modelo de colaboración con la industria en el programa de investigación translacional 9 del IIV implica la evaluación de una vacuna generada por un fabricante incipiente de vacunas, ilustrado por el trabajo del IIV sobre una vacuna contra el cólera de células enteras (WC) inactivadas, administrada por vía oral. La vacuna WC fue trasferida hace dos décadas por el Profesor Jan Holmgren de Suecia a un productor vietnamita (denominado actualmente VaBiotech) [18]. Aunque la vacuna WC oral ha sido utilizada en programas de salud pública en Vietnam durante muchos años, debido a que la autoridad preceptiva nacional vietnamita no está aprobada por la OMS, la vacuna no ha sido utilizada fuera de Vietnam. Los especialistas en producción de vacunas del IIV han trabajado con VaBiotech para mejorar la calidad de producción de la vacuna utilizada en Vietnam con objeto de cumplir con las normas de la OMS. Además, con objeto de permitir el empleo internacional de esta vacuna en países donde el cólera es endémico, el equipo del IIV está transfiriendo la tecnología de producción para esta vacuna a dos productores de países donde el cólera es endémico con autoridades reguladoras nacionales aprobadas por la OMS (India e Indonesia). El IIV está también ayudando a estos productores con el diseño y la realización de los estudios clínicos de fases 2 y 3 necesarios para la aprobación. 
Un cuarto modelo de colaboración implica el desarrollo de un candidato vacunal local en el IIV, con la transferencia subsiguiente del candidato a convertirse en un fabricante de vacunas incipiente y la realización de los estudios clínicos necesarios para la aprobación. Un ejemplo de este modelo estriba en la vacuna conjugada de polisacárido y proteína Vi, desarrollada por el Dr. John Robbins y sus colaboradores en los Institutos Nacionales de la Salud (NIH) de EE.UU., que ha mostrado un efecto altamente protector en un ensayo de fase $2 \mathrm{~b}$ demostrativo del principio en niños vietnamitas que empiezan a andar [19]. No obstante, a pesar de estos datos prometedores ningún productor de vacunas ha desarrollado ésta para tenerla en su surtido de productos. En colaboración con el laboratorio del doctor Robbins, el grupo de proceso y formulación del IIV ha elaborado una nueva vacuna conjugada de toxoide diftérico y Vi y ha emprendido recientemente un programa de transferencia tecnológica y desarrollo clínico para la aprobación de la vacuna en colaboración con un fabricante indio que se ha comprometido a producir la vacuna a precios asequibles para los programas de salud pública en países donde la fiebre tifoidea es endémica.

A partir de estos ejemplos puede apreciarse fácilmente que la realización de ensayos clínicos con vacunas en países en vías de desarrollo es por lo menos uno de los elementos de una estrategia encaminada al logro de acceso a estas vacunas por parte de poblaciones sin recursos de estos entornos. El hecho de compartir los riesgos financieros y de otro tipo inherentes al desarrollo de una vacuna es crucial para asegurar que la vacuna estará disponible para programas de sectores públicos. El IIV se siente afortunado al contar con un personal que posee una amplia gama de aptitudes, incluyendo la investigación del desarrollo y el proceso de vacunas, la transferencia de tecnología vacunal, la realización de ensayos clínicos, la formulación de estrategias reguladoras y las negociaciones comerciales. Esta amplia gama de talentos confiere al IIV un elevado grado de flexibilidad para emprender asociaciones públicas y privadas que complementen sus programas de investigación translacional.

\section{Investigación translacional en vacunas tras la aprobación: Un ejemplo}

Puede parecer extraño que se necesite la investigación translacional para abordar el tercer obstáculo científico para vacunas que han completado ensayos de fase 3 y están ya aprobadas. No obstante, se dispone de una muy larga lista de vacunas que son potencialmente útiles para países en vías de desarrollo, que están aprobadas y se utilizan en países desarrollados, pero que no se emplean profusamente en programas de salud pública de países en vías de desarrollo. En la lista se incluyen, entre otras, la vacuna contra el sarampión, la parotiditis y la rubéola, las vacunas orales de generación más reciente contra la tifoidea y el cólera y las vacunas conjugadas contra el neumococo y HIB. Los motivos de esta disparidad en el uso de estas vacunas entre países desarrollados y en vías de desarrollo son varios y comprenden las limitadas finanzas disponibles para la adquisición de vacunas destinadas a países deprimidos. Otro motivo importante es la escasez de datos en países en vías de desarrollo con respecto a la carga y los costes de las enfermedades destinatarias de estas vacunas, la factibilidad y la aceptabilidad de la introducción de estas vacunas en programas sanitarios de la vida real, los costes y la rentabilidad del uso de las vacunas programáticamente y el impacto preventivo práctico de las vacunas, que cabe esperar en condiciones programáticas.

Un ejemplo de una vacuna infrautilizada que podría generar un gran beneficio para poblaciones de países en vías de desarrollo es la vacuna polisacarídica Vi contra la fiebre tifoidea. A pesar de los datos demostrativos de que la fiebre tifoidea mata cada año a varios cientos de miles de personas en el mundo en vías de desarrollo [20], el uso de vacunas contra las tifoideas en los países en vías de desarrollo es infrecuente. Esta constatación resulta particularmente frustrante si se tiene en cuenta la disponibilidad de dos vacunas de la generación más reciente, que están aprobadas desde hace muchos años: la Ty21a por vía oral y la polisacarídica Vi parenteral. La polisacarídica Vi es especialmente atractiva para países en vías de desarrollo debido a que se administra en una dosis única, es relativamente termoestable y está concedida a numerosos productores de vacunas, tanto compañías multinacionales como compañías del mundo en vías de desarrollo. Cabe destacar por su importancia que varios de estos últimos fabricantes producen esta vacuna económicamente (aproximadamente 0,50 dólares EE.UU. por dosis) y con una gran calidad.

Como parte del programa DOMI, la vacuna polisacarídica Vi fue seleccionada para su introducción acelerada, en función de sus deseables características mencionadas anteriormente, para uso en países en vías de desarrollo. En la tabla 2 se presentan los estudios de investigación realizados con la vacuna Vi y en la fiebre tifoidea en países asociados al DOMI. Los países considerados adoptadores tempranos recibieron el complemento completo de 
estudios que se consideraron necesarios para informar a los responsables de la formulación de políticas sobre la introducción de la Vi.

Los resultados de estos estudios [21-39] demostraron que la fiebre tifoidea es un problema muy importante, especialmente en las poblaciones en estudio del sur y el sudeste asiáticos, que la vacuna Vi podría ser suministrada factiblemente en programas basados tanto en la escuela como en la comunidad, que la rentabilidad de la vacuna Vi la convierten en una 'compra preferente', especialmente en los entornos de elevada incidencia estudiados en el sur y el sudeste asiáticos y que hay una demanda considerable para una vacuna contra la fiebre tifoidea, tanto entre los responsables de la formulación de políticas como los residentes comunitarios en todos los países encuestados.

Basándose en éstos y otros datos, el Grupo de Expertos de la Asesoría Científica de la Organización Mundial de la Salud redactó un proyecto de recomendación para el uso más generalizado de vacunas contra las tifoideas en ámbitos con elevada incidencia de esta enfermedad; al respecto, tanto Pakistán como Indonesia pilotarán el empleo de la inmunización con Vi basada en la escuela en entornos urbanos de incidencia elevada.

\section{Investigación translacional sobre vacunas tras la aprobación: Lecciones aprendidas}

Una gran parte de la investigación translacional realizada por el IIV se ha centrado en la tercera problemática científica: suministro de datos epidemiológicos, clínicos, económicos, conductuales y políticos para responsables de la formulación de políticas en niveles tanto nacional como global, para facilitar la adopción de decisiones racionales sobre la conveniencia de invertir en la introducción de vacunas en programas de salud pública [12]. El IIV ha efectuado este tipo de investigación translacional con vacunas contra infecciones entéricas, infecciones bacterianas invasivas encapsuladas, gripe, tuberculosis e infecciones por flavivirus. Esta investigación se realizó en 22 países de Asia, África y América Latina. En el resto del trabajo describimos varias de las lecciones aprendidas a partir de estos estudios de investigación.

Compromiso de los responsables de la formulación de políticas en el inicio de la investigación

Los responsables locales de la formulación de políticas constituyen un público muy importante con respecto a los resultados de la investigación translacional. En conse- cuencia, uno de los retos principales de estos programas es diseñarlos de modo que respondan a las necesidades de los responsables nacionales de la formulación de políticas en países destinatarios de los resultados.

En su comienzo, el programa DOMI realizó una encuesta de responsables de formulación de políticas y líderes de opinión en Bangladesh, China, India, Indonesia, Pakistán, Tailandia y Vietnam [40]. En conjunto, se efectuaron aproximadamente 200 entrevistas cara a cara, semiestructuradas, con personal de los Ministerios de Sanidad, Ministerios de Finanzas y autoridades preceptivas nacionales, líderes académicos en enfermedades infecciosas y productores locales de vacunas. Los resultados de estas entrevistas mostraron que los responsables de la formulación de políticas precisaban 4 tipos de información para adoptar decisiones acerca de la introducción de nuevas vacunas contra el cólera, shigella y fiebre tifoidea en sus programas de salud pública:

(1) Adquisición de datos epidemiológicos sobre la carga y la distribución de enfermedades en sus poblaciones.

(2) Proyectos de demostración piloto de vacunas aprobadas en condiciones de vida real.

(3) Análisis del impacto económico derivado de la introducción de vacunas en programas de salud pública.

(4) Evaluaciones de las percepciones de la comunidad y los proveedores sobre la enfermedad destinataria y la demanda de una vacuna.

Estos datos guiaron el diseño del programa DOMI y sus estudios componentes de investigación translacional. La encuesta también inició un diálogo en curso entre el programa DOMI y los responsables de formulación de políticas e incrementó el impacto de los resultados de la investigación sobre las decisiones políticas.

\section{Constitución de asociaciones y capacidad}

Si se trata de que la investigación translacional ejerza un impacto sobre la política, debe efectuarse con asociaciones ampliamente inclusivas con todos los interesados importantes. Por ejemplo, el programa DOMI creó con éxito una amplia red de socios, entre los que destacan la Organización Mundial de la Salud, los Ministerios de Sanidad e investigadores locales en países asociados, expertos técnicos internacionales y productores de vacunas tanto internacionales como incipientes.

Una consideración importante para la introducción acelerada de vacunas es realizar la investigación translacional dentro de la infraestructura nacional para investigación existente de los países en vías de desarrollo destinatarios de la investigación. Mientras que la dependencia de los investigadores de los países industrializados y las 
Tabla 2. Matriz de datos elaborada por el programa DOMI para la vacuna Vi contra la fiebre tifoidea

\begin{tabular}{|c|c|c|c|c|c|c|c|}
\hline \multirow[t]{2}{*}{ Tipo de actividad } & \multicolumn{7}{|l|}{ País } \\
\hline & Bangladesh & China & India & Indonesia & Pakistán & Tailandia & Vietnam \\
\hline Estudios prospectivos de carga de fiebre tifoidea & $\times$ & $\times$ & $\times$ & $\times$ & $\times$ & & $\times$ \\
\hline $\begin{array}{l}\text { Metanálisis de los datos de carga de la } \\
\text { enfermedad tifoidea }\end{array}$ & $\times$ & $\times$ & $\times$ & $\times$ & $\times$ & $\times$ & $\times$ \\
\hline $\begin{array}{l}\text { Evaluación de la factibilidad, la aceptabilidad y } \\
\text { el impacto de la inmunización con la vacuna Vi }\end{array}$ & & $\times$ & $\times$ & $\times$ & $\times$ & & $\times$ \\
\hline Coste de los estudios de la enfermedad tifoidea & & $\times$ & $\times$ & $\times$ & $\times$ & & $\times$ \\
\hline Coste de los estudios de suministro de la vacuna Vi & & $\times$ & $\times$ & $\times$ & $\times$ & & $\times$ \\
\hline $\begin{array}{l}\text { Análisis de rentabilidad de la inmunización con } \\
\text { la vacuna } \mathrm{Vi}\end{array}$ & & $\times$ & $\times$ & $\times$ & $\times$ & & $\times$ \\
\hline $\begin{array}{l}\text { Evaluación de la demanda/buena disposición } \\
\text { para pagar por la vacuna Vi }\end{array}$ & & $\times$ & $\times$ & $\times$ & $\times$ & & $\times$ \\
\hline Análisis de la política de introducción de la vacuna Vi & $\times$ & $\times$ & $\times$ & $\times$ & $\times$ & $\times$ & $\times$ \\
\hline
\end{tabular}

instituciones de investigación puede ser útil a corto plazo, es mucho más probable que la investigación sea comunicada a los responsables nacionales de la adopción de decisiones e influir sobre la política si ésta se realiza 'dentro del sistema' y si los países se sienten propietarios de la investigación. Este enfoque de investigación ha sido una piedra angular del programa DOMI.

Un componente esencial de las asociaciones significativas con los profesionales de países en vías de desarrollo es la constitución de capacidad, al respecto de lo cual el programa DOMI ha efectuado considerables inversiones en la formación en los ámbitos de epidemiología, ensayos clínicos, diagnóstico microbiológico, investigación económica y socioconductual, así como en la producción y la regulación de vacunas.

\section{Constitución de una base de datos suficiente a nivel del país}

Tal como ilustran los resultados de la encuesta de responsables de la formulación de políticas del DOMI, dichos responsables precisan de diversos datos multidisciplinarios en sus deliberaciones sobre la introducción de nuevas vacunas. Esto significa a menudo la necesidad de constituir una base de datos completa a nivel del país. Por este motivo, las actividades de investigación translacional del programa DOMI se formularon utilizando un enfoque matricial. En la tabla 2 se presenta una matriz de este tipo para la investigación translacional del programa DOMI sobre la vacuna Vi contra la fiebre tifoidea.
Se comprobó que una forma eficiente de generar estos datos consiste en encajar varios tipos de estudios dentro del mismo proyecto de campo. Tal como se ilustra en la figura 2, los proyectos de demostración de campo de la vacuna polisacarídica Vi contra la fiebre tifoidea del programa DOMI incluía estudios no sólo de la factibilidad, los costes, la aceptabilidad y el impacto protector de la vacuna $\mathrm{Vi}$, sino también la evaluación de la incidencia y los factores de riesgo de la enfermedad tifoidea, los costes de la enfermedad, así como las percepciones de la población acerca de la fiebre tifoidea y la necesidad de una vacuna contra la tifoidea en sus programas.

\section{Estudios multinacionales coordinados}

Aunque los estudios aislados realizados en un país individual pueden ser sin duda de utilidad, los estudios multinacionales coordinados, que generan datos comparativos verosímiles, pueden incrementar considerablemente la utilidad de los estudios de investigación translacionales. Los estudios de fiebre tifoidea multidisciplinarios DOMI que se ilustran en la figura 2 se efectuaron de un modo coordinado, estandarizado, en localizaciones de campo de Hechi, China; Yakarta, Indonesia; Hue, Vietnam; Kolkata, India; y Karachi, Pakistán [37, 39]. En conjunto, en estos estudios se incluyó a más de 200.000 sujetos y la comparación de los datos de los lugares aportó varios conocimientos importantes. Por ejemplo, los estudios revelaron un gradiente de tasas de incidencia de fiebre tifoidea, correspondiendo las tasas máximas a las 
Tabla 3. Incidencia anual de meningitis por Hib en las 3 localizaciones de campo del IVI, 2000-2002

\begin{tabular}{|c|c|c|c|c|c|c|}
\hline \multirow{3}{*}{$\begin{array}{l}\text { Localización } \\
\text { de campo }\end{array}$} & \multirow{3}{*}{$\begin{array}{l}\text { Población } \\
<5 \text { años de } \\
\text { edad }\end{array}$} & \multicolumn{4}{|c|}{ Pacientes con meningitis por Hib } & \multirow{3}{*}{$\begin{array}{l}\text { Hib total } \\
\text { Tasa annual, } \times 10^{-5}\end{array}$} \\
\hline & & \multicolumn{2}{|c|}{ Hib confirmado por cultivo del LCR } & \multicolumn{2}{|c|}{$\begin{array}{l}\text { Hib confirmado por RCP o } \mathrm{AL}(+) / \\
\text { cultivo }(-)^{1}\end{array}$} & \\
\hline & & $\mathrm{n}$ & Tasa annual, $\times 10^{-5}$ & $\mathrm{n}$ & Tasa annual, $\times 10^{-5}$ & \\
\hline Corea & 116.894 & 8 & 3,4 & 6 & 2,6 & 6,0 \\
\hline Vietnam & 94.529 & 13 & 6,9 & 10 & 5,3 & 12,2 \\
\hline China & 168.060 & 3 & 1,1 & 1 & 0,4 & 1,4 \\
\hline
\end{tabular}

LCR = Líquido cefalorraquídeo.

${ }^{1}$ Meningitis por Hib confirmada por la reacción de cadena de polimerasas (RCP) o la aglutinación de látex (AL), pero con cultivo negativo.

localizaciones del sur de Asia (Kolkata y Karachi), la tasa intermedia a la localización del sudeste asiático (Yakarta) y las tasas más bajas a las localizaciones del noreste asiático (Hue y Hechi). En los 3 lugares con las tasas de incidencia máximas (Karachi, Kolkata y Yakarta), la incidencia de fiebre tifoidea fue elevada no sólo en niños de edad escolar, el grupo de edad considerado convencionalmente que presenta el riesgo máximo de fiebre tifoidea, sino también en niños de edad preescolar, una observación con claras implicaciones para las vacunas tifoideas seleccionadas [39].

\section{Dejando que los pedazos caigan donde puedan}

Los estudios de investigación translacional son realizados normalmente por investigadores cuyas expectativas estriban en que los resultados de los estudios motiven la introducción de las vacunas. No obstante, es importante admitir que los estudios 'negativos', que no respaldan fehacientemente la introducción de las vacunas, son tan importantes como los estudios 'positivos'. En el transcurso de los años, la investigación translacional del IIV ha generado ambos tipos de datos. El primer proyecto de investigación multinacional importante del IIV fue un estudio de la carga de meningitis por Hib en niños asiáticos. En este estudio prospectivo, niños menores de 5 años en ubicaciones de Corea, China y Vietnam, cada una con aproximadamente 100.000 niños, fueron seguidos durante períodos de 2 años para estimar la incidencia de meningitis por Hib, utilizando protocolos comunes y métodos diagnósticos exhaustivos y bien estandarizados. Esta investigación se emprendió porque no se habían realizado previamente estudios demográficos, prospectivos, de meningitis por Hib en poblaciones asiáticas y porque existía la sospecha firme de que la meningitis por Hib es-

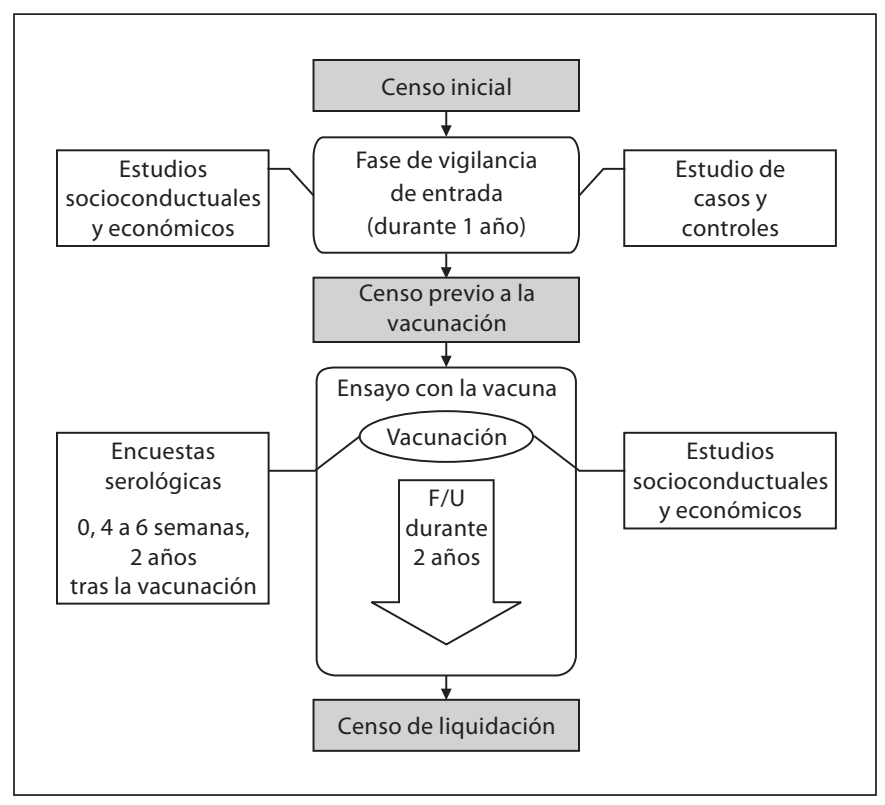

Fig. 2. Estructura de los ensayos de eficacia de la vacuna Vi del programa DOMI.

taba siendo infradiagnosticada en niños asiáticos, dando lugar a la falsa impresión de que esta enfermedad no constituía ningún problema sanitario importante. Para sorpresa de los investigadores, las tasas de incidencia halladas en el estudio del IIV (tabla 3 ) fueron, de hecho, bajas en cada uno de los tres lugares [41-43].

La investigación translacional del IIV de la encefalitis japonesa (EJ) en niños asiáticos proporcionó una experiencia contrastante. Análogamente a la situación de la meningitis por Hib, existía una idea generalizada de que la EJ no constituía ningún problema significativo en los 
países asiáticos próximos al Ecuador a pesar de la ausencia de estudios demográficos, prospectivos. Por otra parte, se disponía de numerosos informes anecdóticos de casos de EJ, confeccionados por médicos de Bali, Indonesia. En consecuencia, los investigadores del IIV, en colaboración con el Ministerio de Salud de Indonesia, emprendieron un estudio prospectivo, durante varios años, de la incidencia de EJ en todos los niños residentes en Bali. De modo impresionante, las tasas de incidencia halladas se situaban entre las más elevadas reportadas en Asia; y estos datos, junto con análisis de la rentabilidad potencial de la vacunación contra la EJ en este entorno, resultó útil para que el Ministerio de Salud adoptara la decisión reciente de introducir la vacunación sistemática contra la EJ de los niños de Bali [44].

\section{Comunicación de los resultados}

La investigación translacional se realiza para informar las decisiones políticas. La publicación de la investigación en revistas apropiadas es importante. Además, la investigación debe comunicarse a los responsables de la formulación de políticas, que pueden no haber leído estas revistas, de un modo oportuno y eficiente. Como ya se ha mencionado, conseguir el compromiso de los responsables de la formulación de políticas al comienzo de la investigación es útil para alentar su interés y continuar el diálogo sobre los datos de la investigación a medida de que éstos vayan apareciendo. Además, es importante comunicar los datos en foros apropiados. Por ejemplo, el programa DOMI ha auspiciado varios simposios especiales y otros foros para comunicar los resultados del programa a los responsables de la formulación de políticas en un nivel global y en niveles regionales (OMS y Alianza GAVI), así como a responsables nacionales de la formulación de políticas de los países asociados al DOMI. Estas sesiones han sido cruciales para la adopción de recientes decisiones políticas por parte de los Ministerios de Salud para pilotear la introducción de la vacuna polisacarídica Vi contra la fiebre tifoidea en programas de Indonesia y Pakistán.

\section{Observaciones en conclusión}

En los años iniciales desde su fundación en 1997, el IIV ha llegado a ser un protagonista importante en el ámbito de la investigación translacional para la introducción de vacunas nuevas en países en vías de desarrollo. Mientras que ya se han acumulado numerosas lecciones de la investigación del IIV y la investigación de por si ha producido un efecto sobre la política de vacunas, la mejora de las estrategias para diseñar y realizar programas de investigación translacional se halla todavía en fase de desarrollo. La reciente disponibilidad de recursos para dichos programas gracias a la Fundación Bill y Melinda Gates y la Alianza GAVI debe incrementar la eficacia y la eficiencia de la investigación, lo cual resulta crucial para países en vías de desarrollo cuyos recursos para nuevas vacunas son escasos y para los cuales la adopción de decisiones políticas correctas es imperativa.

\section{Bibliografía}

1 The Jordan report: accelerated development of vaccines 2007. Bethesda, US Department of Health and Human Services, National Institutes of Health, National Institute of Allergy and Infectious Diseases, 2007, p 157.

2 Tacket CO, Kotloff KL, Rennels MB: Initial clinical evaluation of new vaccine candidates: investigators' perspective of phase I and II clinical trials of safety, immunogenicity, and preliminary efficacy; in Levine $\mathrm{M}$, Kaper J, Rappouli R, Liu M, Good M (eds): New Generation Vaccines. New York, Dekker, 2004, pp 19-28.

3 Clemens J, Naficy A, Rao M, Koo H: Longterm evaluation of vaccine protection: methodological issues for Phase III and IV studies; in Levine M, Kaper J, Rappouli R, Liu M, Good M (eds): New Generation Vaccines. New York, Dekker, 2004, pp 29-48.
4 Deen J, Clemens J: Issues in the design and implementation of vaccine trials in less developed countries. Nat Rev Drug Discov 2006;5:932-940.

5 Vesikari T, Isolauri E, D’Hondt E, et al: Protection of infants against rotavirus diarrhoea by RIT 4237 attenuated bovine rotavirus strain vaccine. Lancet 1984;i:977-981.

6 Hanlon P, Hanlon L, Marsh V, et al: Trial of an attenuated bovine rotavirus vaccine (RIT 4237) in Gambian infants. Lancet 1987;i: 1342-1345.

7 De Mol P, Zissis G, Butzler JP, et al: Failure of live, attenuated oral rotavirus vaccine. Lancet 1986;ii:108.
$\$ 8$ Eskola J, Käyhty H, Takala AK, et al: A randomized, prospective field trial of a conjugate vaccine in the protection of infants and young children against invasive Haemophilus influenzae type b disease. N Engl J Med 1990;323:1381-1387.

-9 Ward J, Brenneman G, Letson GW, et al: Limited efficacy of Haemophilus influenzae type b conjugate vaccine in Alaska native infants. N Engl J Med 1990;323:1393-1401.

10 Mulholland K, Hilton S, Adegbola R, et al: Randomised trial of Haemophilus influenzae type-b tetanus protein conjugate for prevention of pneumonia and meningitis in Gambian infants. Lancet 1997;349:11911197. 
11 Clemens J, Brenner R, Rao M, et al: Evaluating new vaccines for developing countries: efficacy or effectiveness? JAMA 1996;275: 390-397.

12 Clemens J, Jodar L: Introducing new vaccines into developing countries: obstacles, opportunities, and complexities. Nat Med 2005;11(suppl 4):S12-S15.

$\checkmark 13$ Mahoney RT, Maynard JE: The introduction of new vaccines into developing countries. Vaccine 1999;17:646-652.

14 Vesikari T, Matson DO, Dennehy P, et al: Rotavirus Efficacy and Safety Trial (REST) Study Team. Safety and efficacy of pentavalent human-bovine (WC3) reassortant rotavirus vaccine. N Engl J Med 2006;354:2333.

15 Qadri F, Chowdhury MI, Faruque S, et al: Randomized controlled study of the safety and immunogenicity of Peru-15, a live attenuated oral vaccine candidate for Vibrio cholerae $\mathrm{O} 1$ in adult volunteers in Bangladesh. J Infect Dis 2005; 192:573-579.

16 Qadri F, Chowdhury M, Faruque S, et al: Peru-15, a live attenuated oral cholera vaccine is safe and immunogenic in Bangladeshi toddlers and infants. Vaccine 2007;25:231238.

17 Hindle Z, Chatfield SN, Phillimore J, et al: Characterization of Salmonella enteritica derivatives harboring defined aroC and Salmonella pathogenicity island 2 type III secretion system (ssaV) mutations by immunization of healthy volunteers. Infect Immun 2002;70:3457-3467.

- 18 Trach DD, Cam PD, Ke NT, et al: Investigations of the safety and immunogenicity of a new, killed oral cholera vaccine developed in Vietnam. Bull World Health Organ 2002;80: $2-8$.

19 Lin FY, Ho VA, Khiem HB, et al: The efficacy of a Salmonella typhi Vi conjugate vaccine in two-to-five-year-old children. N Engl J Med 2001;344:1263-1269.

20 Crump JA, Luby SP, Minta ED: The global burden of typhoid fever. Bull World Health Organ 2004;82:346-353.

-21 Sur D, von Seidlein L, Manna B, et al: The malaria and typhoid fever burden in the slums of Kolkata, India: data from a prospective community-based study. Trans Royal Soc Trop Med Hyg 2006;100:725733.

-22 Bahl R, Sinha A, Poulos C, et al: Costs of illness due to typhoid fever in an Indian urban slum community: implications for vaccination policy. J Health Popul Nutr 2004;22: 304-310.
23 Zhou WZ, Koo HW, Wang XY, et al: Revaccination with locally-produced Vi typhoid polysaccharide vaccine among Chinese school-aged children: safety and immunogenicity findings. Pediatr Infect Dis J 2007;26: 1001-1005.

24 Acosta CJ, Hong-Hui Y, Ning W, et al: Efficacy of a locally produced, Chinese Vi polysaccharide typhoid fever vaccine during six years of follow-up. Vaccine 2005;23:56185623

25 Chen X, Stanton B, Pach A, et al: Adult perceived prevalence of enteric fever predicts laboratory-validated incidence of typhoid fever in children. J Health Popul Nutr, in press.

26 Kaljee LM, Pham V, Son ND, et al: Trial participation and vaccine desirability for $\mathrm{Vi}$ polysaccharide typhoid fever vaccine in Hue City, Viet Nam. Trop Med Int Health 2007; 12:25-36.

27 Yang HH, Kilgore PE, Yang LH, et al: An outbreak of typhoid fever, Xing-An county, People's Republic of China, 1999: estimation of the field effectiveness of $\mathrm{Vi}$ polysaccharide typhoid vaccine. J Infect Dis 2001;183:17751780.

28 Acosta C, Galindo C, Ochiai RL, et al: The role of epidemiology in the introduction of Vi polysaccharide vaccines in Asia. J Health Popul Nutr 2004;22:240-245.

29 Poulos C, Bahl R, Whittington D, et al: A benefit-cost analysis of typhoid fever immunization programs in an Indian urban slum community: incorporating private market provision of Vi vaccines. J Health Popul Nutr 2004;22:311-321.

30 Yang J, Acosta C, Si G, et al: A mass vaccination campaign targeting adults and children to prevent typhoid fever in Hechi; expanding the use of Vi polysaccharide in Southeast China: a cluster randomized trial. BMC Public Health 2005;18:49-55.

31 Ochiai RL, Wang XY, Yang J, et al: A relative increase in Salmonella paratyphi A rates in Asia: results from surveillance studies in 5 countries. Emerg Infect Dis 2005;11:17641766.

32 Acosta C, Galndo C, Ali M, et al: A multicountry cluster randomized controlled effectiveness evaluation to accelerate the introduction of $\mathrm{Vi}$ polysaccharide typhoid vaccine in developing countries in Asia: rationale and design. Trop Med Int Health 2005;10:1219-1228.

33 Khan MI, Sahito SM, Khan MJ, et al: Enhanced disease surveillance through private health care sector cooperation in Karachi, Pakistan: experience from a vaccine trial. Bull World Health Organ 2006;84:72-77.
34 Khan MI, Ochiai L, Hanza H, et al: Lessons and implications from a mass immunization campaign in squatter settlements of Karachi, Pakistan: an experience from a cluster-randomized, double-blind controlled clinical trial. Trials 2006;7:17.

35 Thiem V, Donovaro-Holliday C, Canh do G, et al: The feasibility of a school-based $\mathrm{Vi}$ polysaccharide vaccine mass immunization campaign in Hue City, central Vietnam: streamlining a typhoid fever preventive strategy. Southeast Asian J Trop Med Public Health 2006;37:515-522.

-36 Agtini M, Ochiai RL, Soeharno R, et al: Introducing $\mathrm{Vi}$ polysaccharide typhoid vaccine to primary school children in North Jakarta, Indonesia, via an existent school-based vaccination platform. Public Health 2006; 120:1081-1087.

37 Ochiai RL, Acosta C, Agtini M, et al: The use of typhoid vaccines in Asia: the DOMI experience. Clin Infect Dis 2007;45(suppl 1):S34S38.

38 Sur D, Ali M, von Seidlein L, et al: Comparisons of predictors for typhoid and paratyphoid fever in Kolkata, India. BMC Public Health 2007;7:289.

39 Ochiai RL, Acosta C, Danovaro-Holliday $\mathrm{MC}$, et al: A multicenter, population-based, prospective surveillance study of typhoid fever in 5 Asian countries: disease burden and implications for control. Bull World Health Organ, in press.

40 DeRoeck D, Nyamete A, Mahoney R, Clemens J: Policymakers' views regarding the introduction of new generation vaccines against cholera, typhoid fever and shigellosis in Asia. Vaccine 2005;23:2762-2774.

41 Anh DD, Kilgore P, Kennedy W, et al: Haemophilus influenzae type b meningitis among children in Hanoi, Vietnam: epidemiologic patterns and estimates of disease burden. Am J Trop Med Hyg 2006;74:509515.

42 Kennedy W, Chang S, Purdy K, et al: Incidence of bacterial meningitis in Asia using enhanced CSF testing: polymerase chain reaction, latex agglutination, and culture. Epidemiol Infect 2007;5:1-10.

43 Kim JS, Jang YT, Kim JD, et al: Incidence of Haemophilus influenzae type b and other invasive diseases in South Korean children. Vaccine 2004;28:3952-3962.

-44 Kari K, Liu W, Gautama K, et al: A hospitalbased surveillance for Japanese encephalitis in Bali, Indonesia. BMC Med 2006;4:8. 